\title{
4 gewinnt: Befestigung, Füllungen, Stumpfaufbau, Unterfülllungen
}

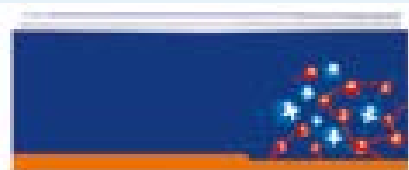

Inoselect
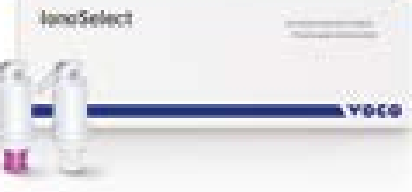

dikationen eines GIZ geeignet ist: Befestigung, Füllungen, Stumpfaufbau, Unterfüllungen. Je nach Wahl des entsprechenden Flüssigkeitskolbens für den Kapselkörper und der dadurch definierten Pulver-Flüssigkeitszusammensetzung in der Applikationskapsel kommt das Material bei verschiedenen Indikationen zum Einsatz. Das Füllungsmaterial in 2 Farben (Universal und Pink) zeichnet sich durch sehr gute physikalische Eigenschaften wie etwa eine hohe Druckfestigkeit, gute Haftung an Schmelz und Dentin sowie hohe Biokompatibilität und Fluoridfreisetzung aus. Es ist u.a. geeignet für Füllungen von nicht okklusionstragenden Klasse-I-Kavitäten, semipermanente Füllungen von Kavitäten der Klassen I und II und Füllungen von Klasse-III- und Klasse-V-Kavitäten. Als zahnfarbenes Befestigungsmaterial ermöglicht es die Befestigung u.a. von metallbasierten Kronen, Brücken, Inlays und Onlays, die Befestigung von hochfesten vollkeramischen Kronen und Brücken sowie die Befestigung von konfektionierten Stahlkronen und Metall-Wurzelstiften bzw. indirekten Metall-StiftStumpfaufbauten. Nach dem Mischen ist das Material sofort einsatzbereit und kann mit einem Applikator eingebracht werden. Die Verarbeitungszeit ist mit mindestens 1,5 min (Füllung, Stumpfbau) bzw. mindestens 2 min (Befestigung) ab Mischbeginn hinreichend lang. IonoSelect erfordert keine Konditionierung der Zahnhartsubstanz, ist feuchtigkeitstolerant und verfügt über eine kurze $\mathrm{Ab}$ bindezeit.

Nach einer Pressemitteilung der VOCO GmbH, Cuxhaven www.voco.de 